\title{
Does physical activity prevent cognitive decline and dementia?: A systematic review and meta-analysis of longitudinal studies
}

Sarah J Blondell ${ }^{1}$, Rachel Hammersley-Mather ${ }^{2}$ and J Lennert Veerman ${ }^{1 *}$

\begin{abstract}
Background: By 2050, it has been estimated that approximately one-fifth of the population will be made up of older adults (aged $\geq 60$ years). Old age often comes with cognitive decline and dementia. Physical activity may prevent cognitive decline and dementia.

Methods: We reviewed and synthesised prospective studies into physical activity and cognitive decline, and physical activity and dementia, published until January 2014. Forty-seven cohorts, derived from two previous systematic reviews and an updated database search, were used in the meta-analyses. Included participants were aged $\geq 40$ years, in good health and/or randomly selected from the community. Studies were assessed for methodological quality.

Results: Twenty-one cohorts on physical activity and cognitive decline and twenty-six cohorts on physical activity and dementia were included. Meta-analysis, using the quality-effects model, suggests that participants with higher levels of physical activity, when compared to those with lower levels, are at reduced risk of cognitive decline, RR $0.65,95 \%$ Cl 0.55-0.76, and dementia, RR $0.86,95 \%$ Cl 0.76-0.97. Sensitivity analyses revealed a more conservative estimate of the impact of physical activity on cognitive decline and dementia for high quality studies, studies reporting effect sizes as ORs, greater number of adjustments ( $\geq 10)$, and longer follow-up time ( $\geq 10$ years). When one heavily weighted study was excluded, physical activity was associated with an $18 \%$ reduction in the risk of dementia (RR 0.82; 0.73-0.91).

Conclusions: Longitudinal observational studies show an association between higher levels of physical activity and a reduced risk of cognitive decline and dementia. A case can be made for a causal interpretation. Future research should use objective measures of physical activity, adjust for the full range of confounders and have adequate follow-up length. Ideally, randomised controlled trials will be conducted. Regardless of any effect on cognition, physical activity should be encouraged, as it has been shown to be beneficial on numerous levels.
\end{abstract}

Keywords: Physical activity, Exercise, Cognitive decline, Dementia, Cognitive impairment, Alzheimer's disease, Cognition

\section{Background}

\section{Cognitive decline}

By 2050, it has been estimated that the world will number 2 billion older adults, aged $\geq 60$ years, or approximately one-fifth $(22 \%)$ of the population [1]. Such a demographic profile will have significant implications on

\footnotetext{
*Correspondence: I.veerman@sph.uq.edu.au

${ }^{1}$ The University of Queensland, School of Population Health, 4006 Herston, Queensland, Australia

Full list of author information is available at the end of the article
}

a multitude of dimensions, including social, economic and health [1]. Individuals aged $\geq 65$ years are over twice as likely as a 20 year old to be afflicted with $\geq 1$ chronic disease, 84 per cent compared to 38 per cent respectively, and other physical and mental disturbances [2]. One particular issue with potentially significant implications for all of the aforementioned factors, is cognitive decline. Research suggests that cognitive decline is associated with the aging process, particularly from age $\geq 50$ years [3]. Whilst some cognitive decline, stemming from reduced 
brain size and plasticity, is normally occurring in most people, not all cognitive decline is considered 'normal' $[4,5]$. The following will outline, in order of severity, 'abnormal' cognitive decline.

\section{Cognitive impairment no dementia (CIND)}

CIND refers to the subjective and objective cognitive decline, which is neither normal nor extreme, experienced by individuals, and which is not sufficient to warrant a diagnosis of dementia [6]. This form of cognitive decline is not debilitating but, nevertheless, causes worry to the sufferer [6]. The criteria for CIND diagnoses are not yet universally agreed upon, but generally relate to a decline in cognitive domains, such as memory, executive function or praxis, that is deemed worse than that of individuals in the same age bracket, of the same sex and educational level [7]. A CIND diagnosis may be made regardless of known underlying mechanisms, such as delirium, substance abuse and psychiatric illness [8]. Approximately one-fifth of Americans aged $\geq 71$ years are thought to suffer from CIND [9]. CIND may be a stand-alone illness or may be a precursor to dementia [9]. A significant proportion, namely half to two-thirds, of those individuals fitting the CIND classification may be further classified as having MCI [10].

\section{Mild cognitive impairment (MCl)}

$\mathrm{MCI}$ is an ever- evolving term; however, it is most widely used to categorise older adults who present with subjective and objective memory impairment that cannot be explained by an underlying neurological or psychiatric ailment, and whose other cognitive functions are generally intact with no impediment to their everyday activities and life [10]. MCI is considered a precursor to dementia and $\mathrm{AD}$.

Neuroimaging of people with MCI has shown early structural changes that are in keeping with what would be expected of AD sufferers [10]. Annually, 10 to 15 per cent of individuals with MCI progress to dementia, as compared to 1 to 2 per cent of healthy adults, and this figure is thought to be on the rise [6]. It is important to note from this that clinically-recognised abnormal cognitive decline represents only an increased risk for dementia, and does not mean that dementia is inevitable; in fact, people may revert back to a previous unimpaired state [11]. The identification of cognitive impairment before full-blown/clinically diagnosed dementia may, however, provide the opportunity for possible preventative measures.

\section{Dementia}

Dementia, like CIND and MCI, is an illness primarily seen and diagnosed in older adulthood; some 90-98 per cent of cases are aged $\geq 65$ years [1]. Dementia is a syndrome that is typified by memory impairment, as well as $\geq 1$ other cognitive deficit [12]. Dementia may be diagnosed if these deficits (1) are incapacitating for the individual, i.e. he/she is no longer able to function in daily tasks; (2) represent a decline from previous functioning; and (3) are not attributable exclusively to a delirium, i.e. a short-onset disturbance in cognition [12]. A number of categories of dementia exist and represent a number of different underlying factors, including dementia with Lewy bodies, vascular dementia( $\mathrm{VaD})$ and Alzheimer's disease $(A D)$, with the latter two sub-types being the most common [13,14].

In 2010, the number of people with dementia was reportedly 35.6 million, with $\mathrm{AD}$ and $\mathrm{VaD}$ accounting for 80 per cent of all clinically diagnosed dementia cases $[1,14]$. There are currently no curative treatment options for sufferers of dementia [15]. Given that, at its extremes, dementia may cause a sufferer to forget key biographical information, including his/her own name and family members, it is not surprising that people with dementia often require some significant degree of care [12]. In 2010, it was estimated that the world-wide economic cost of dementia was US $\$ 604$ billion, the vast majority of which was not provided by the formal sector, but rather by unpaid informal carers, i.e. spouse, child $[1,5]$. Dementia, therefore, is a huge burden for sufferers, their carers, families, friends and the wider community.

Given the high prevalence of dementia, limited treatment options, the costs of the disease to individuals, families and society, and the increased risk of a subset of those with CIND to develop dementia, it is important to explore potential risk and protective factors for both cognitive decline and dementia.

\section{Risk and protective factors for cognitive decline and dementia}

Given the apparent connectedness between cognitive decline and dementia, it seems fitting that they would share many of the same risk and protective factors. Both cognitive decline and dementia share common risk factors. Some of these are amenable to change, others not. Non-modifiable factors associated with both cognitive decline and dementia include age, sex and genetics (particularly APOE genes) $[11,16]$. Whilst it is important to be aware of these, from a preventive point of view, the focus needs to be on measures that are modifiable. Cardiovascular risk factors, including hypertension, diabetes, the metabolic syndrome, obesity and smoking are considered as risk factors for both cognitive decline and dementia $[1,11,15]$. There is suggestive evidence that lifestyle factors may contribute to, or be protective of, cognitive decline and dementia, one such example being physical activity [7]. 


\section{Physical activity as a protective factor for cognitive decline and dementia}

Physical activity is defined as the movement of skeletal muscles, resulting in energy expenditure exceeding the resting state [17]. Physical activity, which encompasses exercise, is different from physical fitness [18]. One can be physically active without necessarily having high aerobic fitness [19]. Physical activity is noted as being beneficial across many domains, including cardiovascular disease, cancer and depression [5].

Much research has been undertaken to assess whether physical activity reduces cognitive decline and prevents dementia. Results from prospective studies have shown mixed results [20,21]. Aarsland et al. 2010, for example, noted that of five studies looking into physical activity and cognitive decline, two reported a significant relationship for both sexes, two reported a significant relationship for females only, and one showed no association [16]. A similar picture emerges for dementia - Aarsland et al. 2010 found that, of 12 studies examining physical activity and dementia, over half did not find statistically significant effects [16]. However, despite individual variation between studies, systematic reviews and meta-analyses of longitudinal studies do lend support to the association between physical activity, cognitive decline and dementia.

Sofi et al. 2011 [20] undertook a systematic review and meta-analysis of prospective studies exploring physical activity and risk of cognitive decline in non-demented individuals. Cognitive decline, or cognitive impairment, was defined as decline in tests of cognitive function at followup (no minimum threshold was specified). Participants with high and low-to-moderate levels of physical activity were compared to those who were sedentary. Included as part of the meta-analyses were 12 prospective studies (15 cohorts) published up until January 2010 [22-33]. All studies, bar one, had participants aged $\geq 65$ years. Both low-to-moderate level (HR 0.65, 95\% CI 0.57- 0.75; p = $<0.001$ ) and high level (HR 0.62, 95\% CI 0.54-0.70; $\mathrm{p}=$ $<0.001$ ) physical activity were shown to be protective of cognitive decline.

Hamer \& Chida 2009 [34] explored the relationship between physical activity and neurodegenerative diseases in healthy adults, at baseline, in meta-analyses of prospective cohort studies. The outcome measure was a diagnosis of dementia or cognitive impairment at followup. Included as part of the meta-analyses were nine studies on dementia and AD (10 cohorts), published up until 2007 [23,35-42]. The highest level of physical activity, when compared with the lowest level, gave a relative risk (RR) of 0.72 (95\% CI $0.60-0.86, \mathrm{p}=<0.001)$ for dementia and 0.55 (95\% CI $0.36-0.84, \mathrm{p}=<0.006)$ for AD.

The following review will summarise results from prospective studies into physical activity and cognitive decline, and physical activity and dementia up to January
2014. The present review is undertaken in view of additional original research in the field, including a number of studies that include objective measures of physical activity. This review also utilises a quality assessment tool to improve the validity of results [43]. Examined in this review are individuals aged $\geq 40$ years who were in good health and/or randomly selected from the community.

\section{Methods}

This paper uses the preferred reporting items for a systematic review and meta-analysis (PRISMA) to ensure accuracy and comprehensiveness [44]. A review protocol was written prior to undertaking the searches. The paper has been registered with PROSPERO, registration number CRD42014008722.

\section{Search strategy}

This review builds on the work of the two most recently published systematic reviews on physical activity and cognitive decline and dementia. Sofi et al. 2011 [20] explored the relationship between physical activity and cognitive decline and Hamer \& Chida 2009 [34] looked at the association between physical activity and dementia. An updated database search retrieved papers published after these reviews. Two modes, therefore, as outlined below, were used to search for relevant literature.

\section{Previous systematic reviews}

Previous systematic reviews on physical activity preventing cognitive decline or dementia were included in the current review. The reference lists of the Sofi et al. 2011 [20] and Hamer \& Chida 2009 [34] articles were examined, and relevant articles sought, providing evidence up until 2010 and 2007, respectively.

\section{Update of database search}

Searches were conducted to identify any relevant studies published since 2007 (the cut-off date for the earliest literature searches from the previous reviews). The PubMed and PsycInfo databases were searched for studies published between 1 January 2007 and 31 December 2013. Terms searched were (("physical activity" OR "exercise") and ("cognitive decline" OR "dementia" OR "cognitive impairment” OR "Alzheimer's disease” OR "cognition")). The search was further limited, using filters available in the databases, to journal articles with subjects aged $\geq 40$ years.

\section{Study selection}

Study selection was limited to those (1) utilising a prospective design; (2) utilising a population-based sample; (3) with a definition of what constitutes cognitive decline or dementia, and a description of the methods used to assess these; (4) with data on baseline physical activity; 
(5) original research articles from dates after identified/ included reviews; and (6) that reported estimates of association between physical activity subgroups and cognitive decline or dementia.

\section{Data extraction}

Key data from the relevant studies were independently extracted and tabulated by two investigators with a master-level degree in health sciences (SJB, RHM). Inclusion of studies was based firstly, on title; secondly, on abstract; or thirdly, on full text. The PRISMA 2009 flow diagram was used as a template for reporting study inclusion [44]. Discussion was undertaken post-extraction by the investigators to resolve any differences of opinion. Where agreement could not be reached, a third investigator with a PhD (JLV), was consulted. Additional file 1: Table S1 presents the extracted information.

\section{Quality assessment}

Included studies were assessed by the two independent reviewers, as described above, for methodological quality with the use of a tool adapted from Singh et al. 2012 [45]. Additional file 2: Table S2 contains the ten items that were used to assess study quality. Each of the items was weighted equally, with a score of one representing a 'yes', and zero a 'no'. Where a paper cited other papers (for further details) the additional papers were sought; however, if the secondary papers did not contain the required information, no additional papers were sought. In such instances the criterion in question was given a score of ' 0 ', for not being stated. If a paper used a standardised measure, and did not report the reliability or validity, a cursory search of Google Scholar (using the measure name and 'validity' or 'reliability') was undertaken. If the required information could not be readily accessed (in the first page of search results), then a ' 0 ' was given. A score (out of ten) was allocated to papers by each reviewer, and any points of difference were discussed until an agreement could be met. Where an agreement could not be met, a third investigator, as above, was consulted. In line with Singh et al. 2012, studies with a score equating to $\geq 70$ per cent were considered as "high quality", and those $<70$ per cent were deemed "low quality" studies [45].

\section{Statistical analysis}

MS Excel with add-in MetaXL [freely available at http:// www.epigear.com/index_files/metaxl.html] was utilised to synthesise the effect sizes from individual studies, with the most fully-adjusted included in the meta-analyses. All effect sizes were reported in their original format, and outputs given as relative risks (RRs). Analyses were carried out on the highest level of physical activity compared to the lowest level of physical activity for cognitive decline and dementia, separately, as this was how most studies reported their results. Heterogeneity was examined in multiple ways. Firstly, it was assessed via the $\mathrm{I}^{2}$ statistic, whereby an $\mathrm{I}^{2}$ statistic of 25 per cent was considered low; 50 per cent, moderate; and 75 per cent, high [46]. Secondly, sensitivity analyses were conducted to address possible sources of heterogeneity. Potential publication bias was assessed with the use of funnel plots.

\section{Results}

\section{Search outputs}

\section{Previous reviews}

Twelve prospective studies (15 cohorts) [22-33] were obtained from the Sofi et al. 2011 [20] systematic metaanalysis on physical activity and risk of cognitive decline, see Figure 1. Nine studies on dementia and AD (10 cohorts) $[23,35-42]$ were derived from the systematic review

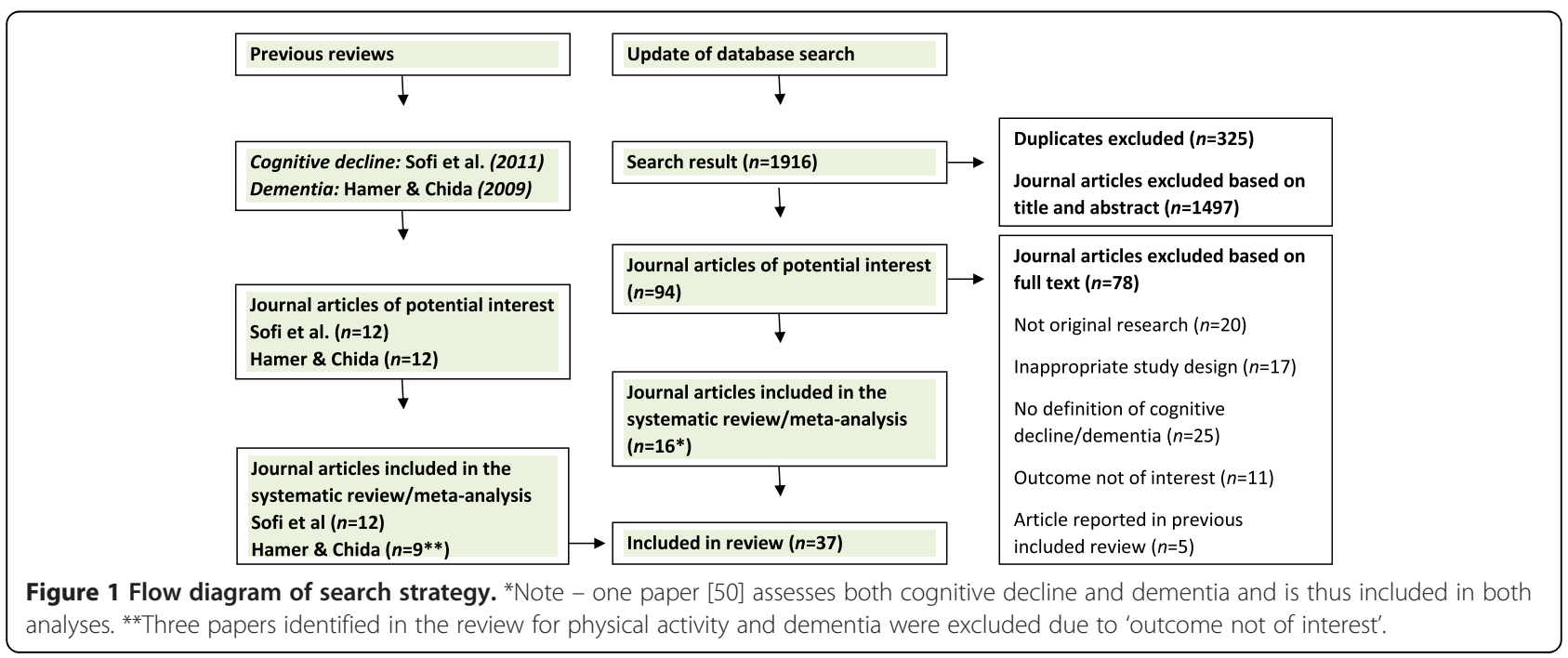


by Hamer \& Chida 2009 [34] on physical activity and neurodegenerative diseases (including dementia).

\section{Database search}

The searches yielded 1916 titles. Of those, 94 potentially relevant papers where identified and full text articles retrieved; see Figure 1. Twenty studies were not original research, seventeen papers had inappropriate study design, no definition of cognitive decline/dementia was given in twenty-five studies, eleven studies reported on outcomes not of interest, and five articles identified were reported in the previous reviews. Sixteen studies met the inclusion criteria and are included in the review [21,47-61]. One small study reported only ORs for the combined condition MCI/AD (Active vs inactive OR: 0.93; 95\% CI 0.45-1.90) [62]. Inclusion of this study did not change the results in either analysis, and was excluded from the analysis/not reported ("outcome not of interest").

\section{Study characteristics}

\section{Cognitive decline}

Assessed were 17 prospective studies (21 cohorts), 12 studies from the Sofi et al. review paper [20] and 5 additional studies identified in the database search [47-51], on physical activity and cognitive decline, see Additional file 1: Table S1. The majority of the cohorts were mixed $(n=9)$; followed by male only $(n=7)$ and female only $(n=5)$. Studies were conducted world-wide, including China, Italy, Netherlands, Australia, Canada, Singapore, Germany, Japan, UK and USA. The sample sizes ranged from 27 to 12303. Follow-up time ranged from 1 to 21 years. Cognitive decline was assessed primarily with the MMSE, 8 cohorts, and the modified MMSE, 5 cohorts. Assessment of physical activity was undertaken via the use of questionnaires, with the exception of one study that used doubly-labelled water and indirect calorimetry [49]. All studies adjusted for confounders, the number of factors ranging from 1 to 18 . Sixteen cohorts controlled for at least age and education, two controlled for age and National Reading Test score, two controlled for age and education only, respectively, and one controlled for neither.

\section{Dementia}

Twenty-one studies on physical activity and dementia (26 cohorts) were examined in relation to physical activity and dementia. Nine studies were from the previous review paper [34] and twelve additional studies were identified in the database search [21,50,52-61] (Additional file 1: Table S1). The majority of the cohorts were mixed sex $(n=18)$; however, some studies reported data for males only $(n=7)$ and females only $(n=1)$. Studies represented countries globally, including Finland, Italy, UK, Australia, Korea, Iceland, Canada, France, Nigeria, the Netherlands, Japan, Hawaii and USA. The sample sizes ranged from 469 to
4945. Follow-up time ranged from 1 to 26 years. Dementia was primarily assessed with the use of a version of the DSM. With the exception of one study that utilised data from an Actigraph [52], physical activity was assessed with the use of questionnaires. Age and education were almost universally adjusted for in all cohorts, age and education $(\mathrm{n}=20)$, age and National Reading Test score $(\mathrm{n}=2)$, age $(n=2)$, education $(n=1)$, and neither $(n=1)$. Other co-variates adjusted for across the studies were broadly classified as demographics, health indicators, gene type and lifestyle factors.

\section{Quality assessment}

A total of eight studies of physical activity and cognitive decline were defined as being of high quality and thirteen studies were deemed as low quality. Fifteen studies on physical activity and dementia were high quality, and the remaining eleven were low quality, see Additional file 3: Table S3.

\section{Meta-analysis}

In light of the heterogeneous nature of the studies included in both analyses (See Additional file 1: Table S1), RRs are derived from a quality effects model. The quality effects model is used when studies differ in relation to study design [43].

\section{Cognitive decline}

Results suggest that participants with higher levels of physical activity, when compared to those with lower levels, are protected against cognitive decline (RR 0.65, 95\% CI 0.55-0.76), as shown in Figure 2. There was moderate heterogeneity between the studies $\left(\mathrm{I}^{2}=52 \%\right)$.

\section{Dementia}

Results suggest that higher levels of physical activity, versus lower levels of physical activity, are associated with a $14 \%$ reduction in the risk of dementia (RR 0.86 , 95\% CI 0.76-0.97), see Figure 3. Heterogeneity between the studies was high $\left(\mathrm{I}^{2}=66 \%\right)$.

\section{Sensitivity analyses}

Sensitivity analyses were performed in view of the nonhomogenous nature of studies in both domains. Studies were grouped by low quality or high quality, ORs or RRs, follow-up time $<10$ or $\geq 10$ years, and number of adjustments $<10$ or $\geq 10$ (see Additional file 4: Table S4). For both cognitive decline and dementia, studies reporting ORs, RR 0.67 and RR 0.89, studies of high quality, RR 0.73 and RR 0.87 , with a greater number of adjustments $(\geq 10)$, RR 0.68 and RR 0.86, and longer follow-up time ( $\geq 10$ years), RR 0.89 and RR 0.86 , respectively, provided more conservative findings. An additional sensitivity analysis was run for the dementia studies by excluding 

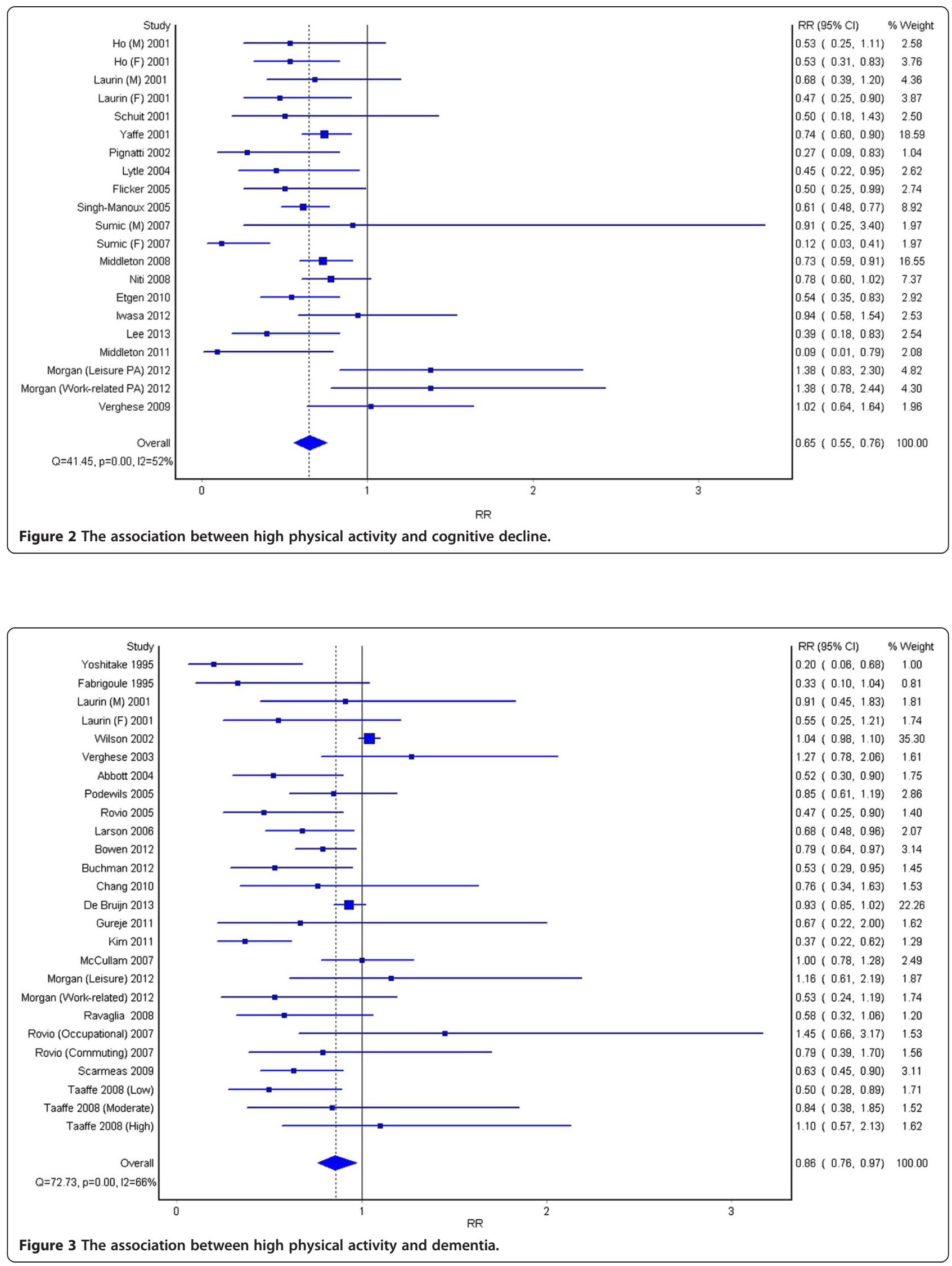
one small study [37] that was weighted heavily in the quality effects model due to its small confidence intervals. Exclusion of this study (Wilson et al. 2002 [37]) strengthened the negative association of physical activity with dementia, RR $0.82(0.73-0.91)$.

\section{Publication bias}

Publication bias was assessed via funnel plots, see Figures 4 and 5. The funnel plot for cognitive decline does not suggest publication bias, but publication bias may have influenced the findings for dementia, where the heavilyweighted study by Wilson (2002) found no effect of physical activity [37].

\section{Discussion}

The aim of this article was to review, update and quantify the association between physical activity, cognitive decline and dementia. The current review shows significant negative associations of physical activity with cognitive decline and dementia, with overall effects of RR 0.65, 95\% CI $0.55-0.76$ and RR 0.86, 95\% CI 0.76-0.97, respectively. The addition of more recent studies to the previous reviews marginally diminished the relationship between physical activity and cognitive decline, and physical activity and dementia. Publication bias may have influenced the results for dementia. Excluding the study by Wilson et al. [37], put the effect of physical activity on dementia as stronger than those reported in the overall findings. This analysis brought results for dementia more in line with those witnessed for cognitive decline. Unlike most authors, Wilson et al. [37] analysed hours of physical activity per week and dementia, not high versus low physical activity. This resulted in (1) more efficient use of the data, and thus (2) more power, (3) a lesser effect, but smaller confidence intervals and, therefore, a weight of approximately 35 per cent in the quality-effects analysis. While this analysis clearly differs from the others, our pre-defined selection criteria, however, provided no grounds to remove the study (despite it being a small study that was weighted heavily in the analysis). On the whole the results do, however, lend support to the notion of a causal relationship between physical activity, cognitive decline and dementia, according to the established criteria for causal inference.

\section{Bradford Hill criteria}

Austin Bradford Hill first established guidelines for causality, which is today often considered the leading method for identifying a causal relationship [63]. It is important to note that Bradford Hill himself did not present these factors as needing to be strictly adhered to, but rather as aids for interpreting associations [64]. The Bradford Hill criteria are (1) strength of association; (2) consistency; (3) specificity; (4) temporality; (5) biological gradient; (6) plausibility; and (7) coherence [65]. While many of these factors are not infallible, the proposed considerations are still useful [65]. In the following paragraphs, we will discuss each in turn.

\section{Association}

This study confirms that there is an association between physical activity and cognitive decline and dementia. In terms of strength, Webb \& Bain 2011 [66] suggest that RR 0.20 should be considered strong, and RR 0.50 considered moderately strong. In line with this, then, the associations established by this review may be considered as low-to-moderate, although the magnitude may in fact

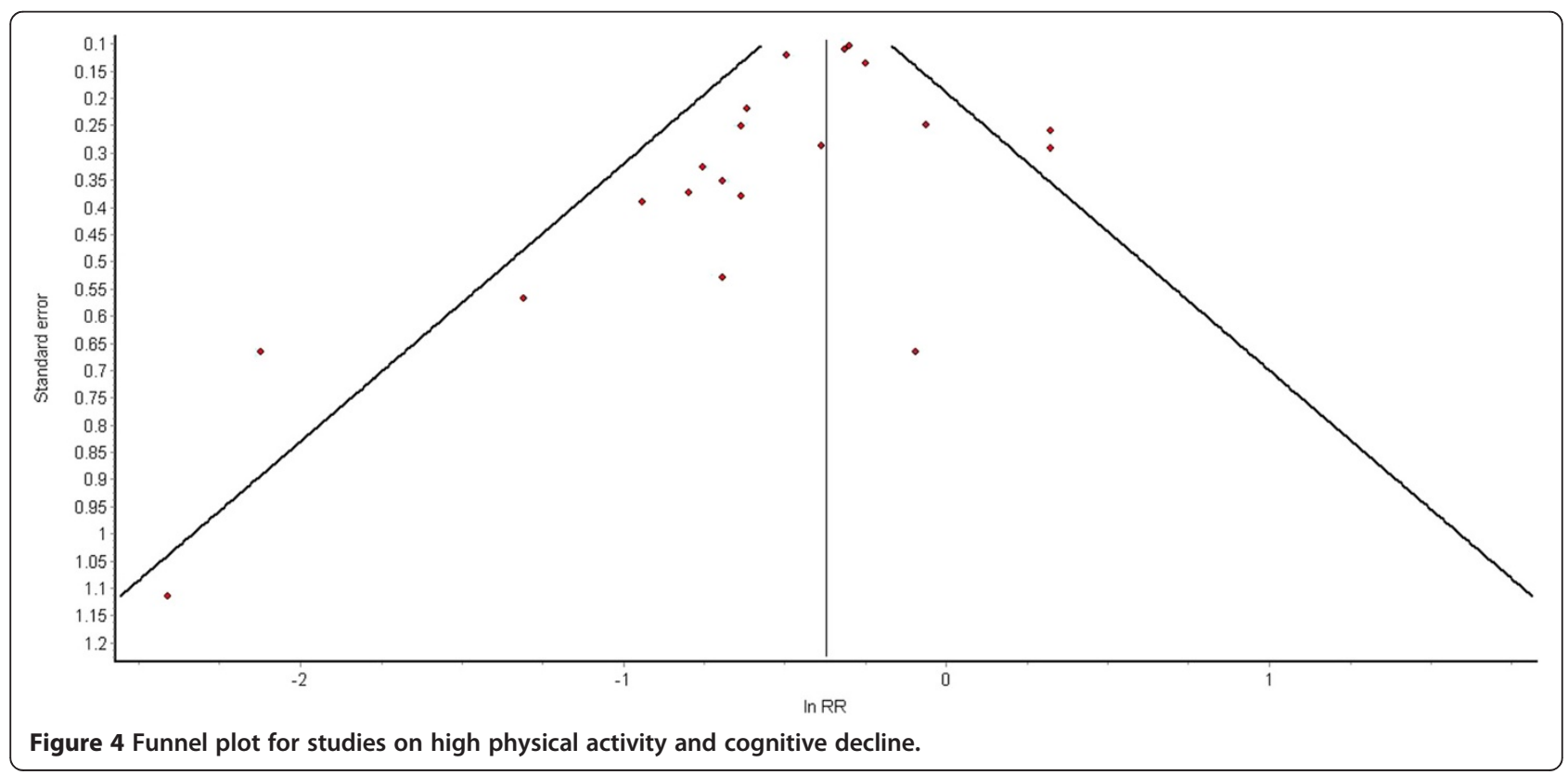




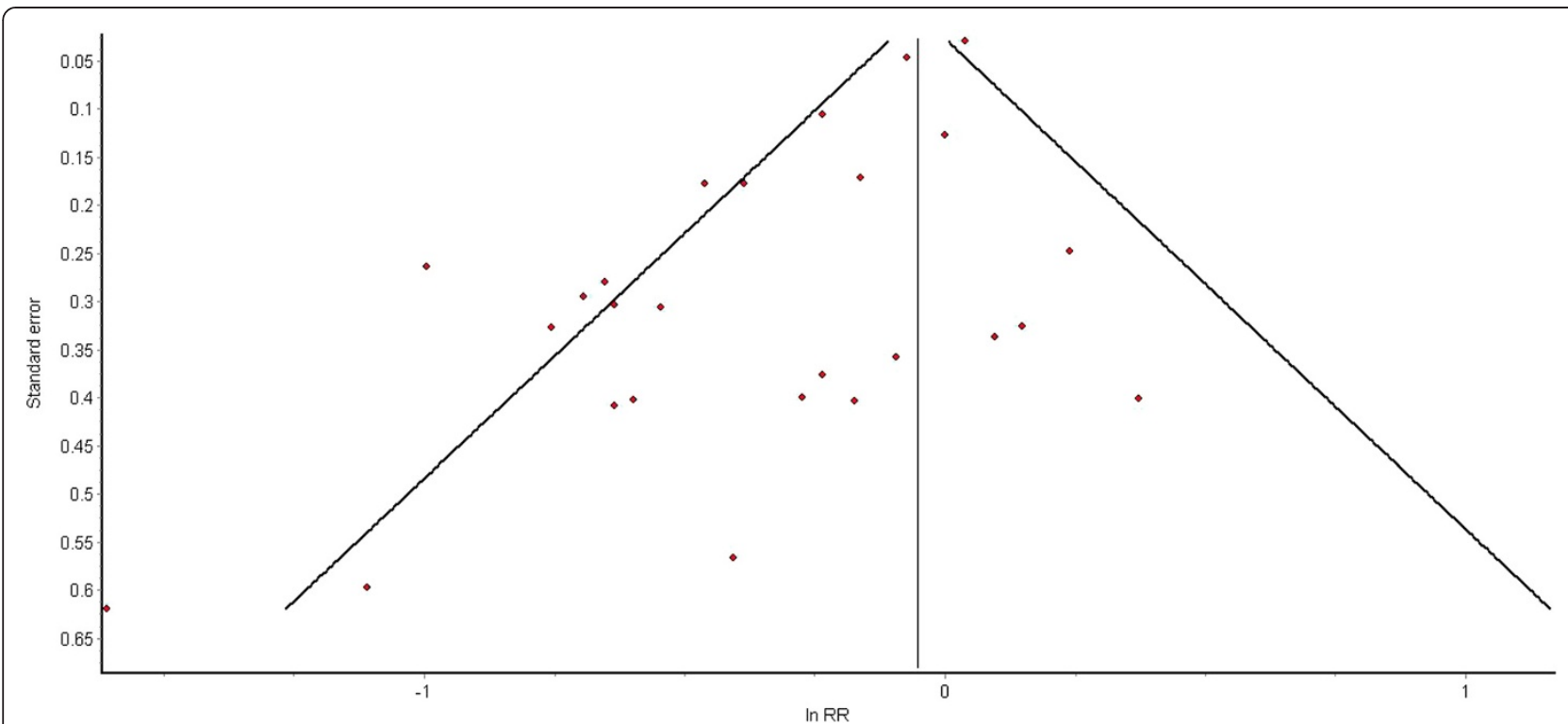

Figure 5 Funnel plot for studies on high physical activity and dementia.

be inaccurate due to several factors, for example publication bias.

Publication bias refers to the under-reporting of results that fail to find significant positive associations [46]. The funnel plot for cognitive decline does not suggest publication bias. However, publication bias may have influenced the findings for dementia, whereby a large number of smaller studies show a larger-than-average effect. Misleading effect sizes may stem also from inadequate control for confounders.

A number of variables have been identified as possible confounders in the relationship between physical activity, cognitive decline and dementia. Scarmeas et al. 2009 [60] identify and control for a comprehensive list of variables in their study; however, a number of other studies included in the current review failed to adequately adjust their data for known confounders. Potential confounders to be controlled for include age, sex, educational level, alcohol consumption, smoking status, depression score, stress, anxiety and cardiovascular disease $[4,16]$. Incomplete adjustment for confounders may inflate the association between physical activity, cognitive decline and dementia and, therefore, control for confounders should be a priority for researchers. One dementia study [42] included in the meta-analysis did not adjust for age or education, both key/plausible confounders; however, exclusion of this study did not alter results significantly (RR: 0.86, 0.76-0.98). Sensitivity analysis into number of adjustments, for example, revealed that those studies which adjusted for a greater number of confounders $(\geq 10)$, as compared to a lesser number $(<10)$, found a smaller protective effect of physical activity on cognitive decline, RR 0.68 (0.51 - 0.91), and dementia, RR 0.86
(0.77 - 0.96). Inadequate control for confounders may also have implications for consistency.

\section{Consistency}

Consistency relates to the reliability of outcomes across different settings, populations, study designs, etc. [64]. Consistency, therefore, inherently relies on sound methodological underpinnings. In the present study, inconsistent protective effects may be traced to the many methodological issues in both domains, as well as to limits in the power of individual studies and, perhaps, real differences in effects across age and other characteristics of the population under study.

Plassman et al. 2010 [7], in their systematic review of observational studies on factors associated with risk for, and possible prevention of, cognitive decline in later life, found cognitive decline to be classified in numerous ways, for example categorical or continuous. However, one test is used predominantly in the literature, the MMSE; in the current review, eight studies utilised the MMSE to assess cognitive decline. The MMSE, a brief 30-item cognitive test, has been the most widely used test to determine an individual's cognitive health, in terms of possible cognitive impairment or dementia. Scores on the MMSE of $<24$ points are considered to be indicative of dementia, and $>3$ point score declines are in line with mild impairment [20]. However, the use of this test is increasingly being challenged, as it is considered to have insufficient scope, and, therefore sensitivity, to identify mild forms of cognitive decline [67]. A modified version of the MMSE, the 3MS, which has an altered scoring system and additional questions, was the second most widely used cognition test across the 
studies in the review [68]. The 3MS is noted as having slightly greater validity and reliability than the MMSE [69]. The screening/assessment of cognitive decline using tests designed to screen for dementia is, however, noted as potentially biasing persons with cognitive decline toward sub-clinical dementia [8]. The utilisation of psychometric tests not specifically designed for assessment of dementia may be more appropriate, and may limit the aforementioned bias.

So, too, it has been noted that physical activity is a somewhat ill-defined term [7]. Physical activity can be described in terms of leisure activity only, all activity, "sweat index", etc. $[4,62]$. These physical activity indicators are then most often assessed via self-reported questionnaire; however, such a subjective method may introduce bias, for example recall and social desirability bias [4]. The physical activity questionnaires utilised in the papers included in the review often failed to provide sufficient detail on activity type, frequency, duration, intensity, and, therefore, limited insights can be drawn of clinical significance. Further, the majority of studies did not report information on the validity or reliability of the measures. Even when physical activity questionnaires are more in depth, however, Middleton et al. 2011 [49] note that older adults may not adequately assess their range of activities, particularly low-intensity physical activity. Inaccuracies in measurement of physical activity, as outlined above, may lead to regression dilution bias, whereby imprecise measurement of the exposure variable may bias a result toward null [70]. There is, therefore, a great need to use objective measures such as accelerometry [4]. Only two studies in the present review utilised an objective measure of physical activity. Buchman [52] used actigraphy in 716 older adults, with a follow-up time of 3.5 years, and found total daily physical activity to be significantly protective for AD (HR 0.53, 95\% CI 0.29-0.95). Likewise, Middleton et al. 2011 [49], in their study of 197 subjects over a follow-up time of 5 to 8 years, found a highly protective effect of physical activity on cognitive decline, as measured via doublylabelled water and indirect calorimetry (high vs low OR: $0.09,95 \%$ CI 0.01-0.79).

\section{Specificity}

Specificity, the notion that one agent causes one disease, is not widely apparent in the physical activity, cognitive decline and dementia domains [65]. As outlined above, numerous factors may contribute to the outcomes of interest. However, the lessened risk for cognitive decline experienced by physically active individuals with the APOE 4 gene, offers strength to the notion of causality, in regard to specificity of susceptibility [66,71]. It must be noted, however, that specificity is not central to establishing a causal relationship, unlike temporality [66].

\section{Temporality}

Reverse causality needs to be considered in the association between physical activity, cognitive decline and dementia. Could cognitive decline and dementia lead to a lack of physical activity, rather than the reverse? It is noted, for example, that individuals with pre-clinical AD may be less inclined to undertake physical activity [1]. To overcome such potential issues around causality, longer follow-up time may be beneficial. The current review had studies with follow-up times ranging from 1 to 21 years for cognitive decline, and 1 to 26 years for dementia. Sensitivity analyses for longer $(\geq 10$ year) and shorter $(<10$ year) follow-up time showed that studies with longer follow-up time found weaker protective effect of physical activity for cognitive decline, RR 0.89 (0.62 - 1.27), and dementia, RR $0.86(0.68-1.11)$. The recent study by Morgan et al. 2012 [50], with a followup time of 16 years, did not lend support to the protective effect of physical activity on cognitive decline or dementia. However, Chang et al. 2010 [53], with the longest follow-up time of all studies, 26 years, found a significant association of physical activity on cognitive decline. Likewise, for dementia, Rovio et al. 2005 [41] found physical activity to be protective over an extended follow-up period (active vs sedentary OR: 0.47, 95\% CI 0.25-0.90). The inconsistencies witnessed in establishing temporality are also mirrored in relation to biological gradient.

\section{Biological gradient}

Dose-response relationships have not been widely reported in the literature. Laurin et al., 2001 [23], for example, witnessed a dose-response relationship in relation to physical activity and cognitive decline in females, but found no dose-response relationship for physical activity and cognitive decline in males, or for either sex in relation to physical activity and dementia. In their meta-analyses, Sofi et al. 2011 [20] found no evidence of a dose-response relationship between physical activity and cognitive decline. Likewise, Hamer \& Chida 2009 [34] did not find a linear-dose response relationship across studies, although such results are not unexpected. A curvilinear relationship, with diminishing returns, seems the most likely association [72]; however, more research in this area is needed. Despite the insufficient evidence for a doseresponse relationship, however, there are mechanisms by which the effects of physical activity on cognitive decline and dementia are biologically plausible.

\section{Plausibility}

A number of hypotheses have been proposed to account for the likelihood of physical activity impacting on cognitive decline and dementia. One relates to enlargement of the cognitive reserve, which results from increased 
brain perfusion stimulated by physical activity; another purports vascular origins, whereby cognitive decline and dementia risk are reduced due to the protective effects of exercise on cardiovascular disease, a risk factor for dementia and, more specifically, atherosclerosis; and another suggests exercise limits stress, which in turn diminishes risk for dementia [73]. These hypotheses are not only plausible, but have often proven accurate in studies [73].

\section{Coherence}

Results from a myriad of sources, namely animal, brain plasticity, brain-imaging, epidemiological, experimental and neuropathological studies, suggest that the above hypotheses, namely cognitive reserve, vascular origins, and stress, have merit [74]. Studies utilising neuroimaging techniques have, for example, found physically active older persons to have greater brain volume than less active older individuals (2-2.5 per cent increase, per physical activity quintile) [74]. In a study of older rats, van Praag et al. 2005 [75] found that those that exercised on a running wheel showed a reversal in declines of neurogenesis (decline in neurogenesis has been implicated in cognitive decline). The proposed causal relationship between physical activity, cognitive decline and dementia is, therefore, in keeping with what is known from research into cognitive functioning more generally. The similarities observed in the RRs for cognitive decline and dementia, in the current review, offer credence to shared underlying mechanisms (which is not surprising, given that the definition of dementia centres on cognitive decline).

\section{Strengths}

Unlike many other studies in the field, the search strategy for the current review was not limited by language barriers, which may have given a more complete picture of the available literature. The search was also independently undertaken by two investigators to ensure thorough and accurate reporting. Evidence included in the review is up to date, and includes the first studies utilising objective measures in this field. Extensive sensitivity analyses were undertaken to adequately address the heterogeneous nature of the studies.

\section{Limitations}

A number of limitations of this study are worth noting. The quality assessment tool, and scoring method, may be considered somewhat arbitrary. It is important to note that the same score could be attained by two very different studies each missing different things. However, there was no basis to differentially weigh study quality aspects. Further limitations are in the available evidence base. The studies used a non-standardised set of methods, which makes it difficult to accurately synthesise the findings. The tendency of authors to compare between groups with high and low (or no) physical activity limits the utility of results. This method, of utilising only data from either end of the spectrum, is a less efficient use of the data compared to analysing hours of physical activity per week, for example. Effect sizes were reported in numerous formats; all outputs, however, were given in relative risks (RRs). This is not ideal; however, there was no means of accurately converting adjusted odds ratios (ORs) to RRs, for example. This approach, of taking ORs to equal RRs, and RRs to equal hazard ratios (HRs), is widely used in the literature, e.g. [16,34].

\section{Conclusions}

The current study provides support for the association between physical activity and cognitive decline and dementia. Although methodological limitations of the current evidence base preclude the drawing of definitive conclusions, a case can be made for a causal interpretation of this association. The implications are statistically and clinically significant. It has been estimated, for example, that 3 million AD cases could be averted globally, with a 10-25 per cent shift in modifiable risk factors, including physical activity [76]. Future research should use objective measures of physical activity, adjust for the full range of known, or likely, confounders, and have adequate followup length. Ideally, randomised-control trials would be conducted; however, implementing constant stimulation of physical activity over a long duration may prove difficult. In the meantime, given that physical activity has been shown to be beneficial across multiple health domains, genders and ages, physical activity should be encouraged regardless of its relationship with cognitive decline and dementia [73].

\section{Additional files}

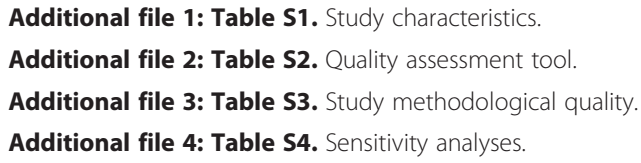

\section{Abbreviations}

AD: Alzheimer's Disease; Cl: Confidence Interval; CIND: Cognitive Impairment No Dementia; DSM: Diagnostic and Statistical Manual of Mental Disorders; HR: Hazard Ratio; ICD: International Classification of Disease; MCl: Mild Cognitive Impairment; MMSE: Mini-Mental State Exam; OR: Odds Ratio; RCT: Randomised Control Trial; RR: Relative Risk; SD: Standard Deviation; VaD: Vascular Dementia.

\section{Competing interests}

The authors declare that they have no competing interests.

\section{Authors' contributions}

SJB participated in the design of the study, extracted data, performed the statistical analysis, drafted the manuscript, and approved the final manuscript. RHM participated in the data extraction and read and approved the final manuscript. JLV conceived the study, and participated in its design and coordination, and read and approved the final manuscript. 


\section{Authors' information}

SJB has a BBeSc, GDiplH, and MPH. RHM has a BIntSt, GradDiplntIDev, MA (Research) and MHID (Health and International Development). JLV has a MD, $\mathrm{MPH}$ and $\mathrm{PhD}$

\section{Acknowledgments}

The authors wish to thank Dr Jan Barendregt for his input regarding the meta-analyses. Dr Mark Griffin for his help relating to the statistical analysis. Thanks also go to Josephine Blondell for editing the manuscript.

\section{Author details}

${ }^{1}$ The University of Queensland, School of Population Health, 4006 Herston, Queensland, Australia. ${ }^{2}$ Counselling and Health at Student Services, University of Southern Queensland, 4350 Toowoomba, Queensland, Australia.

Received: 1 April 2014 Accepted: 8 May 2014

Published: 27 May 2014

\section{References}

1. World Health Organization: Dementia: A Public Health Priority. 2012. whqlibdoc.who.int/publications/2012/9789241564458_eng.pdf.

2. Mortazavi SS, Mohammad K, Ardebili HE, Beni RD, Mahmoodi M, Keshteli $\mathrm{AH}$ : Mental disorder prevention and physical activity in Iranian elderly. Int J Prev Med 2012, 3(Suppl 1):S64-S72.

3. Angevaren M, Aufdemkampe G, Verhaar HJJ, Aleman A, Vanhees L: Physical activity and enhanced fitness improve cognitive function in older people without known cognitive impairment, a Cochrane Systematic Review. Parkinsonism Relat Disord 2008, 14:S44.

4. Miller DI, Taler V, Davidson PSR, Messier C: Measuring the impact of exercise on cognitive aging: methodological issues. Neurobiol Aging 2012, 33(3):622.e629-622.e643.

5. Ahlskog JE, Geda YE, Graff-Radford NR, Petersen RC: Physical exercise as a preventive or disease-modifying treatment of dementia and brain aging. Mayo Clin Proc 2011, 86(9):876-884

6. Rosenberg PB, Johnston D, Lykestos CG: A clinical appraoch to mild cognitive impairment. Am J Psychiatr 2006, 163(11):1884-1890.

7. Plassman BL, Williams JW Jr, Burke JR, Holsinger T, Benjamin S: Systematic review: factors associated with risk for and possible prevention of cognitive decline in later life. Ann Intern Med 2010, 153(3):182-193.

8. Ritchie K, Touchon J: Mild cognitive impairment: conceptual basis and current nosological status. Lancet 2000, 355(9199):225-228.

9. Lautenschlager NT, Cox K, Cyarto EV: The influence of exercise on brain aging and dementia. Biochim Biophys Acta (BBA) - Mol Basis Dis 2012, 1822(3):474-481.

10. Chertkow H, Nasreddine Z, Joanette $Y$, Drolet V, Kirk J, Massoud F, Belleville $\mathrm{S}$, Bergman $\mathrm{H}$ : Mild cognitive impairment and cognitive impairment, no dementia: Part A, concept and diagnosis. Alzheimers Dement 2007, 3(4):266-282.

11. Molinuevo JL, Valls-Pedret C, Rami L: From mild cognitive impairment to prodromal Alzheimer disease: A nosological evolution. Eur Geriatr Med 2010, 1(3):146-154

12. American Psychiatric Association: Diagnostic and Statistical Manual of Mental Disorders: DSM-IV-TR ${ }^{\oplus}$. Washington, DC: American Psychiatric Pub; 2000.

13. Sosa-Ortiz AL, Acosta-Castillo I, Prince MJ: Epidemiology of dementias and Alzheimer's disease. Archives of Medical Research 2012, 43(8):600-608.

14. Lin F, Friedman E, Quinn J, Chen DG, Mapstone M: Effect of leisure activities on inflammation and cognitive function in an aging sample. Arch Gerontol Geriatr 2012, 54(3):e398-e404.

15. Cyarto EV, Lautenschlager NT, Desmond PM, Ames D, Szoeke C, Salvado O, Sharman MJ, Ellis KA, Phal PM, Masters CM, Rowe CC, Martins RN, Cox KL: Protocol for a randomized controlled trial evaluating the effect of physical activity on delaying the progression of white matter changes on MRI in older adults with memory complaints and mild cognitive impairment: The AIBL Active trial. BMC psychiatry 2012, 12(1):167.

16. Aarsland D, Sardahaee FS, Anderssen S, Ballard C, the Alzheimer's Society Systematic Review: Is physical activity a potential preventive factor for vascular dementia? A systematic review. Aging Ment Health 2010, 14(4):386-395.

17. Fuchs R: Physical Activity and Health. In International Encyclopedia of the Social \& Behavioral Sciences. Edited by Neil JS, Paul BB. Oxford: Pergamon; 2001:11411-11415.
18. Tseng CN, Gau BS, Lou MF: The effectiveness of exercise on improving cognitive function in older people: a systematic review. J Nurs Res 2011, 19(2):119-131.

19. Benedict C, Brooks SJ, Kullberg J, Nordenskjold R, Burgos J, Le Greves M, Kilander L, Larsson EM, Johansson L, Ahlstrom H, Lind L, Schioth HB: Association between physical activity and brain health in older adults. Neurobiol Aging 2013, 34(1):83-90.

20. Sofi F, Valecchi D, Bacci D, Abbate R, Gensini GF, Casini A, Macchi C: Physical activity and risk of cognitive decline: a meta-analysis of prospective studies. J Intern Med 2011, 269(1):107-117.

21. Bowen ME: A prospective examination of the relationship between physical activity and dementia risk in later life. Am J Health Promot 2012, 26(6):333-340.

22. Ho SC, Woo J, Sham A, Chan SG, Yu AL: A 3-year follow-up study of social, lifestyle and health predictors of cognitive impairment in a Chinese older cohort. Int J Epidemiol 2001, 30(6):1389-1396.

23. Laurin D, Verreault R, Lindsay J, MacPherson K, Rockwood K: Physical activity and risk of cognitive impairment and dementia in elderly persons. Arch Neurol 2001, 58(3):498-504.

24. Schuit AJ, Feskens EJ, Launer LJ, Kromhout D: Physical activity and cognitive decline, the role of the apolipoprotein e4 allele. Med Sci Sports Exerc 2001, 33(5):772-777

25. Yaffe K, Barnes D, Nevitt M, Lui LY, Covinsky K: A prospective study of physical activity and cognitive decline in elderly women: women who walk. Arch Intern Med 2001, 161(14):1703-1708.

26. Pignatti F, Rozzini R, Trabucchi M: Physical activity and cognitive decline in elderly persons. Arch Intern Med 2002, 162(3):361-362.

27. Lytle ME, Vander Bilt J, Pandav RS, Dodge HH, Ganguli M: Exercise level and cognitive decline: the MoVIES project. Alzheimer Dis Assoc Disord 2004, 18(2):57-64.

28. Flicker L, Almeida OP, Acres J, Le MT, Tuohy RJ, Jamrozik K, Hankey G, Norman P: Predictors of impaired cognitive function in men over the age of 80 years: results from the Health in Men Study. Age Ageing 2005, 34(1):77-80

29. Singh-Manoux A, Hillsdon M, Brunner E, Marmot M: Effects of physical activity on cognitive functioning in middle age: evidence from the Whitehall II prospective cohort study. Am J Public Health 2005, 95(12):2252-2258.

30. Sumic A, Michael YL, Carlson NE, Howieson DB, Kaye JA: Physical activity and the risk of dementia in oldest old. J Aging Health 2007, 19(2):242-259

31. Middleton L, Kirkland S, Rockwood K: Prevention of CIND by physical activity: different impact on VCl-ND compared with $\mathrm{MCl}$. J Neurol SC 2008, 269(1-2):80-84.

32. Niti M, Yap KB, Kua EH, Tan CH, Ng TP: Physical, social and productive leisure activities, cognitive decline and interaction with APOE-epsilon 4 genotype in Chinese older adults. Int Psychogeriatr 2008, 20(2):237-251.

33. Etgen T, Sander D, Huntgeburth U, Poppert H, Forstl H, Bickel H: Physical activity and incident cognitive impairment in elderly persons: the INVADE study. Arch Intern Med 2010, 170(2):186-193.

34. Hamer M, Chida Y: Physical activity and risk of neurodegenerative disease: a systematic review of prospective evidence. Psychol Med 2009, 39(1):3-11.

35. Yoshitake T, Kiyohara Y, Kato I, Ohmura T, Iwamoto H, Nakayama K, Ohmor S, Nomiyama K, Kawano H, Ueda K, Sueishi K, Tsuneyoshi M, Fujishima M: Incidence and risk factors of vascular dementia and Alzheimer's disease in a defined elderly Japanese population: the Hisayama Study. Neurology 1995, 45(6):1161-1168.

36. Fabrigoule $C$, Letenneur $L$, Dartigues JF, Zarrouk M, Commenges D, Barberger-Gateau P: Social and leisure activities and risk of dementia: a prospective longitudinal study. J Am Geriatr Soc 1995, 43(5):485-490.

37. Wilson RS, Bennett DA, Bienias JL, Aggarwal NT, Mendes De Leon CF, Morris $M C$, Schneider JA, Evans DA: Cognitive activity and incident $A D$ in a population-based sample of older persons. Neurology 2002, 59(12):1910-1914.

38. Verghese J, Lipton RB, Katz MJ, Hall CB, Derby CA, Kuslansky G, Ambrose AF, Sliwinski $M$, Buschke $H$ : Leisure activities and the risk of dementia in the elderly. N Engl J Med 2003, 348(25):2508-2516.

39. Abbott RD, White LR, Ross GW, Masaki KH, Curb JD, Petrovitch H: Walking and dementia in physically capable elderly men. JAMA 2004, 292(12):1447-1453. 
40. Podewils LJ, Guallar E, Kuller LH, Fried LP, Lopez OL, Carlson M, Lyketsos CG: Physical activity, APOE genotype, and dementia risk: findings from the Cardiovascular Health Cognition Study. Am J Epidemiol 2005, 161(7):639-651.

41. Rovio S, Kareholt I, Helkala E, Viitanen M, Winblad B, Tuomilehto J, Soininen $\mathrm{H}$, Nissinen A, Kivipelto M: Leisure-time physical activity at midlife and the risk of dementia and Alzheimer's disease. Lancet Neurol 2005, 4:705-711.

42. Larson EB, Wang L, Bowen J, McCormick W, Teri L, Crane P, Kukull W: Exercise Is Associated with Reduced Risk for Incident Dementia among Persons 65 Years of Age and Older. Ann Intern Med 2006, 144:73-81.

43. Doi SA, Thalib L: A quality-effects model for meta-analysis. Epidemiology 2008, 19(1):94-100.

44. Moher D, Liberati A, Tetzlaff J, Altman DG: Preferred reporting items for systematic reviews and meta-analyses: the PRISMA statement. Ann Intern Med 2009, 151(4):264-269.

45. Singh A, Uijtdewilligen L, Twisk JW, Van Mechelen W, Chinapaw MJ: Physical activity and performance at school: a systematic review of the literature including a methodological quality assessment. Arch Pediatr Adolesc Med 2012, 166(1):49-55.

46. Livingston JD, Boyd JE: Correlates and consequences of internalized stigma for people living with mental illness: a systematic review and meta-analysis. Soc Sci Med 2010, 71(12):2150-2161

47. Iwasa H, Yoshida $Y$, Kai I, Suzuki T, Kim H, Yoshida H: Leisure activities and cognitive function in elderly community-dwelling individuals in Japan: A 5-year prospective cohort study. J Psychosom Res 2012, 72(2):159-164.

48. Lee S, Yuki A, Nishita Y, Tange C, Kim H, Kozakai R, Ando F, Shimokata H: Research relationship between light-intensity physical activity and cognitive function in a community-dwelling elderly population-an 8-year longitudinal study. J Am Geriatr Soc 2013, 61(3):452-453.

49. Middleton LE, Manini TM, Simonsick EM, Harris TB, Barnes DE, Tylavsky F, Brach JS, Everhart JE, Yaffe K: Activity energy expenditure and incident cognitive impairment in older adults. Arch Intern Med 2011. 171(14):1251-1257

50. Morgan GS, Gallacher J, Bayer A, Fish M, Ebrahim S, Ben-Shlomo Y: Physical activity in middle-age and dementia in later life: findings from a prospective cohort of men in Caerphilly, South Wales and a meta-analysis. J Alzheimers Dis 2012, 31(3):569-580.

51. Verghese J, Cuiling W, Katz MJ, Sanders A, Lipton RB: Leisure activities and risk of vascular cognitive impairment in older adults. J Geriatr Psychiatry Neurol 2009, 22(2):110-118.

52. Buchman AS, Boyle PA, Yu L, Shah RC, Wilson RS, Bennett DA: Total daily physical activity and the risk of $A D$ and cognitive decline in older adults. Neurology 2012, 78(17):1323-1329.

53. Chang M, Jonsson PV, Snaedal J, Bjornsson S, Saczynski JS, Aspelund T, Eiriksdottir G, Jonsdottir MK, Lopez OL, Harris TB, Gudnason V, Launer L: The effect of midlife physical activity on cognitive function among older adults: AGES-Reykjavik Study. J Gerontol A: Biol Med Sci 2010, 65(12):1369-1374.

54. De Bruijn RF, Schrijvers EM, De Groot KA, Witteman JC, Hofman A, Franco $\mathrm{OH}$, Koudstaal PJ, Ikram MA: The association between physical activity and dementia in an elderly population: the Rotterdam Study. Eur J Epidemiol 2013, 28(3):277-283.

55. Gureje $\mathrm{O}$, Ogunniyi A, Kola L, Abiona T: Incidence of and risk factors for dementia in the Ibadan Study of Aging. J Am Geriatr Soc 2011, 59(5):869-874

56. Kim JM, Stewart R, Bae KY, Kim SW, Yang SJ, Park KH, Shin IS, Yoon JS: Role of BDNF val66met polymorphism on the association between physical activity and incident dementia. Neurobiol Aging 2011, 32(3):551 e555-512.

57. McCallum J, Simons LA, Simons J, Friedlander Y: Delaying dementia and nursing home placement: the Dubbo study of elderly Australians over a 14-year follow-up. Ann N Y Acad Sci 2007, 1114:121-129.

58. Ravaglia G, Forti P, Lucicesare A, Pisacane N, Rietti E, Bianchin M, Dalmonte E: Physical activity and dementia risk in the elderly: findings from a prospective Italian study. Neurology 2008, 70(19 Pt 2):1786-1794.

59. Rovio S, Kareholt I, Viitanen M, Winblad B, Tuomilehto J, Soininen H, Nissinen A, Kivipelto M: Work-related physical activity and the risk of dementia and Alzheimer's disease. Int J Geriatr Psychiatry 2007 22(9):874-882

60. Scarmeas N, Luchsinger JA, Schupf N, Brickman AM, Cosentino S, Tang MX, Stern Y: Physical activity, diet, and risk of Alzheimer disease. JAMA 2009, 302(6):627-637.
61. Taaffe DR, Irie F, Masaki KH, Abbott RD, Petrovitch H, Ross GW, White LR: Physical activity, physical function, and incident dementia in elderly men: the Honolulu-Asia Aging Study. J Gerontol A: Biol Med Sci 2008, 63(5):529-535

62. Sattler C, Erikson Kl, Torro P, Shroder J: Physical fitness as a protective factor for cognitive impairment in a prospective population-based study in Germany. J Alzheimers Dis 2011, 26:709-718.

63. Ward AC: The role of causal criteria in causal inferences: Bradford Hill's "aspects of association". Epidemiol Perspect Innov 2009, 6:2.

64. Rothman KJ, Greenland S, Lash TL: Modern Epidemiology. Philadelphia, PA: Lippincott Williams \& Wilkins; 2008

65. Hofler M: The Bradford Hill considerations on causality: a counterfactual perspective. Emerg Themes Epidemiol 2005, 2:11.

66. Webb P, Bain C: Essential Epidemiology: An Introduction for Students and Health Professionals. Cambridge: Cambridge University Press; 2010.

67. Jacova C, Kertesz A, Blair M, Fisk JD, Feldman HH: Neuropsychological testing and assessment for dementia. Alzheimers Dement 2007, 3(4):299-317.

68. Tombaugh TN: Test-retest reliable coefficients and 5-year change scores for the MMSE and 3MS. Arch Clin Neuropsychol 2005, 20(4):485-503.

69. McDowell I, Kristjansson B, Hill GB, Hébert R: Community screening for dementia: The Mini Mental State Exam (MMSE) and modified Mini-Mental State Exam (3MS) compared. J Clin Epidemiol 1997, 50(4):377-383.

70. MacMahon S, Peto R, Cutler J, Collins R, Sorlie P, Neaton J, Abbott R, Godwin J, Dyer A, Stamler J: Blood pressure, stroke, and coronary heart disease. Part 1, Prolonged differences in blood pressure: prospective observational studies corrected for the regression dilution bias. Lancet 1990, 335(8692):765-774

71. Woodard JL, Nielson KA, Sugarman MA, Smith JC, Seidenberg M, Durgerian S, Butts A, Hantke N, Lancaster M, Matthews MA: Lifestyle and genetic contributions to cognitive decline and hippocampal integrity in healthy aging. Curr Alzheimer Res 2012, 9(4):436.

72. Pate RR, Pratt M, Blair SN, Haskell WL, Macera CA, Bouchard C, Buchner D, Ettinger W, Heath GW, King AC, Kriska A, Leon AS, Marcus BH, Morris J, Paffenbarger RS, Patrick K, Pollock ML, Rippe JM, Sallis J, Wilmore JH: Physical activity and public health. A recommendation from the Centers for Disease Control and Prevention and the American College of Sports Medicine. JAMA 1995, 273(5):402-407.

73. Fratiglioni $L$, Paillard-Borg S, Winblad B: An active and socially integrated lifestyle in late life might protect against dementia. Lancet Neurol 2004 , 3(6):343-353.

74. Ho AJ, Raji CA, Becker JT, Lopez OL, Kuller LH, Hua X, Dinov ID, Stein JL, Rosano C, Toga AW, Thompson PM: The effects of physical activity, education, and body mass index on the aging brain. Hum Brain Mapp 2011, 32(9):1371-1382.

75. Van Praag H, Shubert T, Zhao C, Gage FH: Exercise enhances learning and hippocampal neurogenesis in aged mice. J Neurosci 2005, 25(38):8680-8685.

76. Erickson Kl, Weinstein AM, Lopez OL: Physical activity, brain plasticity, and Alzheimer's disease. Arch Med Res 2012, 43(8):615-621.

doi:10.1186/1471-2458-14-510

Cite this article as: Blondell et al:: Does physical activity prevent cognitive decline and dementia?: A systematic review and meta- analysis of longitudinal studies. BMC Public Health 2014 14:510. 